Article

\title{
Action at a Distance in Quantum Theory
}

\author{
Jerome Blackman ${ }^{1,2}$ \\ 1 Syracuse University, Syracuse, NY 13244, USA; E-Mail: jblackman@alum.mit.edu; \\ Tel.: +315-699-8730 or $+754-220-5502$. \\ 27005 Lakeshore Rd. Cicero, NY 13039, USA
}

Academic Editor: Palle E.T. Jorgensen

Received: 8 March 2015 / Accepted: 22 April 2015 / Published: 6 May 2015

\begin{abstract}
The purpose of this paper is to present a consistent mathematical framework that shows how the EPR (Einstein. Podolsky, Rosen) phenomenon fits into our view of space time. To resolve the differences between the Hilbert space structure of quantum theory and the manifold structure of classical physics, the manifold is taken as a partial representation of the Hilbert space. It is the partial nature of the representation that allows for action at a distance and the failure of the manifold picture.
\end{abstract}

Keywords: action at a distance; EPR; measurement theory

\section{Introduction}

In many books and articles on quantum theory two different statements appear. The first is that quantum theory is the most accurate theory in the history of physics and the second is that it is an incomplete theory. Both of these statements are true, but the incompleteness assertion usually does not refer to the fact that not all questions are answerable at any given time, which is true of all interesting theories, but that quantum theory is a very uncomfortable fit with our usual picture of what kind of space we live in, namely some sort of three or four dimensional manifold. We propose to show that it may be more reasonable to think of Hilbert space as our natural space and the problem arising in reconciling that with our instinct to consider otherwise is the source of some confusion. Further, the failure of our space model to fully reflect the complexity of Hilbert space is the source of the feeling of incompleteness and the puzzling aspect of EPR phenomena. We shall try to show how special relativity fits in but the main emphasis is showing its limitations and analyzing one specific example of action at a distance. 
The problem of action at a distance is intimately connected to the measurement problem $[1,2]$ which is, in turn, connected to the problem of the reversibility of time in the fundamental equations of the theory. This is then connected to the problem of whether information can be lost which has been a problem of great interest to the theoreticians of black holes in recent years [3]. For us it becomes a question of whether the time development is always governed by a unitary group including the measurement process.

The measurement problem has been of great importance and great controversy since the earliest days of quantum theory. Roger Penrose [4] devotes a considerable part of his book to this problem including a conjecture that the solution might lie in a deeper understanding of quantum gravity. The reason this problem has appeared so troublesome is that two early errors have been perpetuated over many years that confused the problem. The first was the observation that a measurement seemed to be a projection in Hilbert space which clearly was not reversible. The problem here was that the argument was correct if the relevant Hilbert space was the space of the particle alone but not if the correct space was the space of the particle and the measuring device. The other error was the observation that the equations of quantum mechanics were deterministic and the results of the measurement were probabilistic. However a measurement always involves a macroscopic device with a large number of constituent particles which at the quantum level are likely to be in a different configuration at each measurement.

With these observations it was possible to define what a measurement should be (Equation (3.2) for the finite spectrum case and Equation (3.6) for the infinite spectrum case) in terms of a condition on the Hamiltonian. Both these conditions are just the statement that a measurement should not introduce any new spectrum during the measurement process. The importance of this theory is that since it describes a measurement as just an interaction between the particle and the measuring device there is no reason to believe it is not just the result of a unitary group of transformations. Article [1] developed the theory for the finite spectrum case and article [2] extended the analysis to the infinite spectrum in the case of measuring the position of an electron. This is an "explanation" of the collapse of the wave function which bedeviled so many students over the years. In the early days of quantum theory the collapse of the wave function was treated as a serious problem but in more recent times it has often been treated as an axiom of the theory. This allows us in this article to apply the unitary properties of the measurement of position to the treatment of action at a distance.

Article [1] breaks down into two distinct parts. The first is the analysis of the physics and the second is a mathematical theorem giving the needed probability results. Here we give a quick recapitulation of the first part. We present a clearer presentation of [2] for reasons stated below.

In the next section we present an analysis of the relation between the Hilbert space approach and the space-time approach of classical physics. The result is a space-time that is only a mathematical model of the Hilbert space model which is faulty because it is based on the information available before the advent of modern technology. The conclusion is that, for example, the Minkowski space of special relativity is just not applicable in the case of action at a distance during the time interval between the splitting of the particle and its reconstitution by a measurement. 


\section{Hilbert Space and Space-Time}

We start with the quantum picture in which an isolated system can be described by a state vector $|q\rangle$ in a Hilbert space $H$, and the development in time is obtained by the action of a one parameter unitary group $U(\tau)$ operating on $|q\rangle$. Of course this program has only worked in simple cases and the most important developments have taken place using the Lagrangian rather than the Hamiltonian formalism but this is the generally accepted picture. The question is then: "How do we get from this picture to the space-time we all grew up with?"

Start with the Schrödinger picture. We then have a three dimensional Euclidean space $E^{3}$ and the complex valued functions whose square is integrable, commonly known as $L^{2}$. Suppose we have $n$ independent objects with Hilbert spaces $H_{1}, H_{2}, \ldots, H_{n}$. Then the space of the collection is Equation (2.1):

$$
H=H_{1} \otimes H_{2} \otimes \ldots \otimes H_{n} .
$$

If $n=1$ and $H_{1}$ represents a macroscopic object such as a pebble we know the wave function in $E^{3}$ will be localized and the Schrödinger equation turns into the Newton equations of motion. If $n=2$ and we are dealing with another pebble (still independent of the first) then the same result holds but the joint wave function should be represented in $L^{2} \otimes L^{2}$ i.e., as a function on $E^{3} \otimes E^{3}$ which is inconvenient for our program. Therefore we will project the wave function on $E^{3} \otimes E^{3}$ onto the first of these spaces and call the result $\widehat{E^{3}}$ which is a three dimensional Euclidean space now with two separate and disjoint wave functions defined on it. More specifically if $f_{1}\left(x_{1}, y_{1}, z_{1}\right) \otimes f_{2}\left(x_{2}, y_{2}, z_{2}\right)$ is the tensor in $L^{2} \otimes L^{2}$ then we map it into $f_{1}\left(x_{1}, y_{1}, z_{1}\right)+f_{2}\left(x_{1}, y_{1}, z_{1}\right)$ in $L^{2}$. At this point we are no longer in the domain of quantum theory but rather in the ordinary space with two disjoint macroscopic objects. All the other terms in Equation (2.1) are treated the same way. As one might conjecture $\widehat{E}^{3}$ will be our model for ordinary space but one further step remains. Let us suppose that there is one more term in Equation (2.1), say $H_{n+1}$ which represents the quantization of an electromagnetic field on $\widehat{E^{3}}$. We are now ready to argue our thesis. To do this we introduce the observer in the form of a state in the Hilbert space

$$
H=H_{1} \otimes H_{2} \otimes \ldots \otimes H_{n+1} \otimes H_{n+2}
$$

Here $H_{n+2}$ represents the observer who might be a geometer from the Age of Pericles or even some distant ancestor of our species. His interaction with the states in $H_{1} \otimes H_{2} \otimes \ldots \otimes H_{n+1}$ is of a quantum nature but the information that reaches his brain is limited by his senses. Nevertheless what reaches the central nervous system is a bunch of chemical and electric signals and it is the job of the brain to interpret those signals into some sort of useful model. We are presenting $\widehat{E^{3}}$ as that model. As noted above for macroscopic objects it gives a very usable model for most mechanical computations up to fairly modern times. The binding force at the molecular level would provide measuring sticks and the path of light would provide straight lines from which the geometer could invent Euclidean geometry. (We'll get to relativity in a moment) Time would appear as the parameter of the unitary group $U(\tau)$. All the non-interacting macroscopic objects would appear as localized non-overlapping "things" in $\widehat{E^{3}}$ 
The weakness of the model becomes apparent when work at the quantum level comes into play. An electron or even a neutron does not behave anything like a pebble. When the wave function of a particle is broken into two parts and the parts separated by some distance, the manifold structure comes into question. In a Riemannian manifold the distance between two points has meaning but when one of the points has no particular location a problem arises. At any rate, it is not surprising that a model based on very limited information should break down when further information is added. What is noteworthy is that in our model "action at a distance" is an example of the ordinary model breaking down at the macroscopic level.

We know from Einstein that the finite speed of light leads to the fact that observers in different inertial systems will differ in their perception of time. Above we specified that an observer would take his time as being the same as the parameter of the unitary group $U(\tau)$. If the two observers have a relative velocity $v$ then their times are related by $d t^{\prime}=d t \sqrt{1-v^{2}}=\tilde{v} d t$ where $\tilde{v}=\sqrt{1-v^{2}}$. Hence $t^{\prime}=\tilde{v} t+k$ where $k$ is a constant. Therefore the unitary group in terms of the second observer's time is related to that of the first observer by

$$
U\left(t^{\prime}\right)=U(\tilde{v} t+k)=U(\tilde{v} t) U(k)
$$

It is easy to see that if $U(t)$ is unitary so is $U(\tilde{v} t) . U(k)$ acts as a translation in time and can be ignored. It follows from this construction that the second observer will see the action of $U$ the same way but on a different time scale and that there are no preferred observers. There are some interesting questions that arise from this construction that are for further work. In particular we are not getting into the question of relativistic field theory here.

\section{Action at a Distance}

We are going to analyze in detail an example of action at a distance which will use the work above but also leans heavily on the theory of quantum measurement presented in [1] and [2] so we will outline the relevant results.

Suppose we have an observable of the particle with a finite number of eigenvalues $p_{1}, \ldots, p_{n}$ and corresponding eigenvectors $\left|p_{i}\right\rangle$ for $i=1, \ldots, n$. Let $H_{p}$ be the $\mathrm{n}$ dimensional Hilbert space of the observable and $H_{p, i}$ the one dimensional Hilbert space spanned by $p_{i}$ so that $H_{p}$ is the direct sum of these subspaces. Define $H_{m}$ as the Hilbert space of the measuring device. What every quantum measurement demands is that the measurement of any eigenstate yields that eigenstate. The measurement takes place in the space $H_{m} \otimes H_{p}$.. Let $\psi$ be a subset of the integers $1,2 \ldots, \mathrm{n}$ and let $H_{p, \psi}$ be the subspace of $H_{p}$ whose basis is the set of $\left|p_{i}\right\rangle$ with $i \in \psi$. The condition on the Hamiltonian $\widehat{H}$ that it be the generator of the unitary group of a measurement of the observable is (by definition) that

$$
\widehat{H}\left(H_{m} \otimes H_{p, \psi}\right) \subseteq\left(H_{m} \otimes H_{p, \psi}\right) \text { for all } \psi
$$

It is easy to show that this is equivalent to

$$
\widehat{H}\left(H_{m} \otimes H_{p, \mathrm{i}}\right) \subseteq\left(H_{m} \otimes H_{p, \mathrm{i}}\right) \text { for all i. }
$$


This is perhaps easier to verify and perhaps to visualize.

It is worth noting that if $\widehat{H_{0}}$ is any linear operator on $H_{m}$ and $\widehat{I_{p}}$ is the identity on $H_{p}$ then $\widehat{H_{0}} \otimes \widehat{I}_{p}$ satisfies Equation (3.2) (as well as Equation (3.6) below). The unitarity question is more speculative. In the literature [3] the model for quantum theory assumes the unitarity of the time development with the exception of the measuring process. As described in Section 2 we can now drop this exception. If the theory is correct it means the assumption of unitary development can be extended to all quantum transformations which is essential to this paper.

For simplicity of notation we can consider $H_{m}$ to have a denumerable orthonormal basis $\left|q_{j}\right\rangle$. Statistics comes into the theory because the measuring device is a macroscopic object which implies that its interaction with the particle cannot be predicted and therefore while the unitary group will describe a curve on the surface of the unit sphere in $H_{m} \otimes H_{p}$ it will change for each measurement. We will not bother to introduce another index but it should be kept in mind. Suppose the initial state of the combined system is $\sum_{j} b^{j}\left|q_{j}\right\rangle \otimes \sum_{i} a^{i}\left|p_{i}\right\rangle$. Then during the measurement we obtain

$$
U(t) \sum_{i, j} b^{j} a^{i}\left|q_{j}\right\rangle \otimes\left|p_{i}\right\rangle=\sum_{i, j} a^{i, j}(t)\left|q_{j}\right\rangle \otimes\left|p_{i}\right\rangle
$$

where $a^{i, j}(0)=b^{j} a^{i}$. Setting $\sum_{j} a^{i, j}(t)\left|q_{j}\right\rangle=\left|g^{i}(t)\right\rangle$ this becomes

$$
U(t) \sum b^{j} a^{i}\left|q_{j}\right\rangle \otimes\left|p_{i}\right\rangle=\sum_{i}\left|g^{i}(\mathrm{t})\right\rangle \otimes\left|p_{i}\right\rangle
$$

Taking the norm of Equation (3.4) and remembering that it is on the unit sphere of $H_{m} \otimes H_{p}$ we have

$$
\left.\sum_{i}|| g^{i}(t)\right\rangle\left.\right|^{2}=1
$$

We consider the terms $\left|g^{j}(t)\right\rangle$ as generalized coefficients of the eigenvectors $\left|p_{i}\right\rangle$.

If we consider $\left.\left.\left.\left(|| g^{1}(t)\right\rangle\right|^{2}, \ldots|| g^{n}(t)\right\rangle\left.\right|^{2}\right)$ as a point in $E^{n}$ then we have a curve on the simplex $\sum_{1}^{n} x_{i}=1$ with $x_{i}>0$. If at some time $t$ one of these terms takes the value 0 Equation (3.1) guarantees that that term remains 0 at all subsequent times. In [1] it is shown that a wide variety of probability measures in the space of all possible paths leads to the path reaching the various vertices of the simplex with the Bohr probabilities. It can be seen from this model that a quantum measurement is a type of random filtering process in which different parts of the initial spectrum are eliminated. The generalization to the infinite spectrum case is briefly described in [1] and applied to the measurement of the position of an electron in [2]. Unfortunately due to the carelessness of the author as a proof reader some errors and unfortunate choices of notation appear. For example in Equation (1) of that paper a string of symbols indicating tensor multiplication $(\otimes)$ appear and every other of these should be symbols for direct sums $(\oplus)$. However we now have a simpler approach and we will apply it directly in an example of action at a distance. The basic idea of how to go from the finite spectrum to the infinite is rather simple and uses the fact that the result of any measurement can only be one of a finite number of possibilities. The trick is to lump all spectral values together that lead to the same 
measurement. One of the benefits of this approach is that the analog of Equation (3.1) or its equivalent Equation (3.2) shows the added difficulty of making finer measurements.

One of the examples of action at a distance described in [3] is that of a photon that is passed through a partially silvered mirror which splits the photon into two parts which are then separated. The experimenter then discovers that finding the photon is at one position guarantees the opposite result at the other position. However the work above is highly dependent on the particle's position being described as a sum of terms enumerated by the eigenvalues of the position operator i.e., as a wave function and the photon is a poor choice since it is equally likely to be anywhere. Getting around this is probably possible but it will be simpler to use a massive particle such as an electron instead. Suppose then that an electron is sent through two slits and each slit is backed up by electron traps which are then separated by some distance. Suppose two electron detectors are located in the $x, y$ plane with the two traps located above them. Let $A_{i}$ for $i=1,2$ be the disjoint areas in the $x, y$ plane below the two traps. We divide $E^{3}$ into three parts $S_{i}$ for $i=1,2,3$. For $i=1,2$ let $S_{i}=\left\{(x, y, \mathrm{z}) \mid(\mathrm{x}, \mathrm{y}) \in \mathrm{A}_{i}\right.$ and $\left.z>0\right\}$ and let $S_{3}=E^{3}-\left(S_{1} \cup S_{2}\right)$. The two detectors are assumed to have the property that they will signal the presence of the electron if almost all of the wave function arrives in its domain. (The "almost all" is just a way of taking into account that the mathematics allows for the misbehavior of a set of measure zero.)

To relate the sets $S_{i}$ to the work above note that the eigenfunctions of the position operator are the delta functions of $E^{3}$ so that $\left\{S_{i} \mid i=1,2,3\right\}$ is a division of all the eigenfunctions into 3 sets. Let $L_{k}^{2}$ for $k=1,2,3$ be the set of all $L^{2}$ functions whose support lies in $S_{k}$. Then $L^{2}=L_{1}^{2} \oplus L_{2}^{2} \oplus L_{3}^{2}$. Now choose an orthonormal basis for each of these three subspaces. Let $\left\{\varphi_{i}^{k} \mid i=1,2, \ldots.\right\}$ be an orthonormal basis for $L_{k}^{2}$. Then the union of these three sets of bases is an orthonormal basis for $L^{2}$. If $\widehat{H}$ is to be the Hamiltonian for the unitary group of the measuring process the analog of the conditions Equations (3.1) or (3.2) is that

$$
\widehat{H}\left(H_{m} \otimes L_{k}^{2}\right) \subseteq H_{m} \otimes L_{k}^{2} \text { for } k=1,2,3 .
$$

Equation (3.6) guarantees that if at some time in the measuring process a point is in $H_{m} \otimes L_{k}^{2}$, for example, it remains there at all subsequent times.

Now let's return to the measuring process. Suppose that the measurement process starts at $\tau_{0}$. Before that the measuring device and the particle are not interacting. Suppose the state of the particle at $\tau_{0}$ is described by the wave function

$$
\phi=\sum_{j} \alpha^{j} \phi_{j}^{1}+\sum_{j} \beta^{j} \phi_{j}^{2}+\sum_{j} \gamma^{j} \phi_{j}^{3}
$$

We note for use below that $\iiint_{E_{1}}|\phi|^{2} d x d y d z=\sum_{j}\left|\alpha^{j}\right|^{2}$ with similar equations for $E_{2}$ and $E_{3}$. If the initial state of the measuring device is $\sum_{i} a^{i}\left|q_{i}\right\rangle$ then the initial state of the whole system is described by $Q=\sum_{i} a^{i}\left|q_{i}\right\rangle \otimes\left(\sum_{j} \alpha^{j} \phi_{j}^{1}+\sum_{j} \beta^{j} \phi_{j}^{2}+\sum_{j} \gamma^{j} \phi_{j}^{3}\right)$. If $U(\tau)$ is the unitary group generated by $i \widehat{H}$ then for $\tau>\tau_{0}$ the state of the system is described by an expression of the form 


$$
U(\tau) Q=\sum_{i, j} a_{1}^{i, j}(\tau)\left|q_{i}\right\rangle \otimes \phi_{j}^{1}+\sum_{i, j} a_{2}^{i, j}(\tau)\left|q_{i}\right\rangle \otimes \phi_{j}^{2}+\sum_{i, j} a_{3}^{i, j}(\tau)\left|q_{i}\right\rangle \otimes \phi_{j}^{3}
$$

Setting $\sum_{i} a_{k}^{i, j}(\tau)\left|q_{i}\right\rangle=g_{k}^{j}(\tau)$ which is in $H_{m}$ we get

$$
U(\tau) Q=\sum_{j} g_{1}^{j}(\tau) \otimes \phi_{j}^{1}+\sum_{j} g_{2}^{j}(\tau) \otimes \phi_{j}^{2}+\sum_{j} g_{3}^{j}(\tau) \otimes \phi_{j}^{3}
$$

Suppose that for some $\tau_{0}$ the first term is 0 . Then from Equation (3.6) and the linearity of $\widehat{H}$ we see that it will be zero for $\tau \geq \tau_{0}$. The problem has now been reduced to the case of the discrete spectrum. We assume the norm of the starting point at $\tau=0$ is 1 so taking the norm in Equation (3.8) and using the orthonormal properties of the basis elements $\alpha_{k}^{i}$ we have

$$
1=\sum_{j}\left\|\mathrm{~g}_{1}^{j}\right\|^{2}+\sum_{j}\left\|g_{2}^{j}\right\|^{2}+\sum_{j}\left\|g_{3}^{j}\right\|^{2}
$$

Again we can consider three terms in Equation (3.9) as the coordinates of a point on the two dimensional simplex in 3 space $\sum_{1}^{3} x_{i}=1$ with $x_{i} \geq 0$.

Before we proceed further it is useful to step back for a moment and consider the simpler case of an ordinary electron which is to be projected onto the $z=0$ plane to see whether it is above $A_{1}$ or $A_{2}$. If we apply the probability theory of reference [1] the result will be that one of the terms in Equation (3.10) will have the value 1 at the end of the measurement and the other two will have the value 0 . Suppose the first term becomes 1 and the others become 0 . Then the ordinary interpretation is that the electron was located above the first detector. According to our measurement theory the measuring device (in this case the two detectors and the projection device) reduced the wave function to one whose support was in $S_{1}$ and that this occurred with probability $\iiint_{E_{1}}|\phi|^{2} d x d y d z$ where $\phi$ was the original wave function. But the interesting thing here is that the third term becomes 0 . This was caused by the random behavior of the interaction between the detectors and the wave function even though the wave function could be spread over a vast region of space and the detectors are small localized devices. This is an essential point which helps understanding the final result which is the analysis of the separated electron problem. It illustrates why the unitary properties of $U(\tau)$ is essential. It is also an explanation of the 'collapse of the wave function' in terms of the measurement theory of [1] and [2]. Now let us return to the electron with the split wave function and we imagine the two detectors are widely separated.

Suppose that the experimenter applies a test to see whether the electron is above the first detector. If the first term in Equation (3.10) has the value 1 as a result then the other two terms are each zero and any attempt to find the electron there will fail. If the first term becomes 0 then the sum of the other two is one. If a subsequent test is made at the second detector the second term becomes 1 or 0 depending on whether the electron is there or the electron was lost. This is the formal explanation of action at a distance in this instance. In terms of the discussion of Section 2 we note that as soon as the wave function is split the model $\widehat{E^{3}}$ is no longer valid. From that point in time until the localization of the electron by a measurement the concept of a metric space is no longer valid and the problem should be thought of as one lying in Hilbert space. Fortunately the theory of measurement is completely in 
Hilbert space terms consisting mainly of the condition Equation (3.6) and the requirement that the state of the measuring device at the end of the measurement should be different for each of the possible results.

\section{Conclusions}

The idea that our familiar metric space is a secondary construction with limited validity is rather radical, but the fact that the metric depends on the relative velocity of two observers, or the existence of EPR effects, are hints of this idea. An argument against it is that the construction of Hamiltonians in elementary cases uses a metric, but these cases all seem to involve electromagnetic effects where the metric is built in. To the extent that unified theory is correct, the same probably applies to the weak and strong forces as well. At any rate, if the theory is useful it opens a door to new investigations.

It was pointed out above that the essential idea in the analysis of action at a distance is the possibility of extending the general assumption of unitary transformations to include the measuring process. Without unitarity the whole process fails. It is very likely that this cannot be accomplished without going into field theory.

\section{Conflicts of Interest}

The author declares no conflict of interest.

\section{References}

1. Blackman, J.; Hsiang, W.T. Why probability appears in quantum theory? Phys. Essays 2013, 26, 34-39.

2. Blackman, J. On wave particle duality. Phys. Essays 2013, 26, 347-349.

3. Susskind, L. The Black Hole War; Little, Brown and Company: New York, NY, USA, 2008.

4. Penrose, R. The Road to Reality; Alfred A. Knopf: New York, NY, USA, 2006.

(C) 2015 by the author; licensee MDPI, Basel, Switzerland. This article is an open access article distributed under the terms and conditions of the Creative Commons Attribution license (http://creativecommons.org/licenses/by/4.0/). 